\title{
Electronic Transport through a Quantum Dot Coupled to Non-Collinear Ferromagnetic Electrodes: The Kondo Regime
}

\author{
R. ŚWIRKOWICZ ${ }^{a}$, M. WAWrZYNIAK ${ }^{b}$, J. BARNAŚ $^{b, c}$ \\ AND M. WILCZYŃSKI ${ }^{a}$ \\ ${ }^{a}$ Faculty of Physics, Warsaw University of Technology \\ Koszykowa 75, 00-662 Warszawa, Poland \\ ${ }^{b}$ Department of Physics, Adam Mickiewicz University \\ Umultowska 85, 61-614 Poznań, Poland \\ ${ }^{c}$ Institute of Molecular Physics, Polish Academy of Sciences \\ Smoluchowskiego 17, 60-179 Poznań, Poland
}

\begin{abstract}
Linear and non-linear conductance of quantum dots attached to magnetic leads is considered theoretically in the Kondo regime. The considerations are based on the non-equilibrium Green function formalism and the relevant equation of motion method. Splitting of the Kondo anomaly due to coupling of the dot to ferromagnetic electrodes, and its dependence on magnetic configuration of the system and on the lead's spin polarization is studied numerically.
\end{abstract}

PACS numbers: $73.23 . \mathrm{Hk}, 73.63 . \mathrm{Kv}$

\section{Introduction}

The Kondo effect in electronic transport through quantum dots (QDs) is of current interest and recent experimental observations are in agreement with theoretical predictions [1]. Spin polarization of conduction electrons in ferromagnetic electrodes (leads) gives rise to additional features of the Kondo phenomenon [2], which have not been observed in the case of non-magnetic leads. Spin dependent coupling of the dot to ferromagnetic electrodes leads to spin splitting of the dot level, which can be taken into account via an effective exchange field $B_{\text {ex }}$ acting on the dot. The level splitting $\Delta$ is then proportional to $B_{\text {ex }}[3]$. As a result the zero-bias anomaly in conductance is also split and considerably suppressed. In 
the present paper we study the Kondo assisted transport through a QD coupled to ferromagnetic electrodes in both linear and non- linear response regimes. In a general case, magnetic moments of the leads are non-collinear and form an angle $\theta$.

\section{Model}

We consider a single level quantum dot described by the standard Anderson Hamiltonian. The dot is connected via tunnel barriers to ferromagnetic electrodes, which are treated as reservoirs of non-interacting and spin polarized electrons. Tunnelling processes between the QD and electrodes with non- collinear magnetic moments are described by the general Hamiltonian $H_{\mathrm{T}}=\sum_{k \beta s \sigma}\left(W_{k \beta}^{s \sigma} a_{k \beta s}^{+} d_{\sigma}+\right.$ h.c.). Elements of the non-diagonal matrix $\hat{W}_{k \beta}$ depend on the angle $\theta$ and describe electron tunnelling from the state with spin $\sigma$ in the dot to the electron band with spin $s(s=+$ or -$)$ in the electrode $\beta(\beta=\mathrm{L}, \mathrm{R})$. These matrix elements are related to the coupling strengths $\Gamma_{s}^{\beta}$. The latter parameters can be written as $\Gamma_{s}^{\beta}=\Gamma_{0}\left(1+s p_{\beta}\right)$, where $p_{\beta}$ is a spin polarization factor of electrons at the Fermi level in the lead $\beta$, and the coupling parameter $\Gamma_{0}$ is constant in the electron band of width $D$ and zero otherwise.

Electron transport through the system is studied with the non-equilibrium Green function formalism, based on the equation of motion method and the decoupling procedure appropriate for the Kondo regime [4]. Electric current is expressed in terms of the retarded $G^{\mathrm{r}}$ (advanced $G^{\mathrm{a}}$ ) and lesser $G^{<}$Green functions. $G^{\mathrm{r}}$ and $G^{\mathrm{a}}$ are calculated from the equation of motion, written in the form of the Dyson equation. As the appropriate self-energy is determined from the Dyson equation, $G^{<}$can be found from the Keldysh formula using the $\mathrm{Ng}$ ansatz [5]. Such an approach enables description of electron transport in both linear and non-linear transport regimes.

\section{Results and discussion}

Numerical calculations have been performed in the limit of strong electron correlations, $U \rightarrow \infty$, and for symmetrical systems described by the following parameters: $\Gamma_{0}=0.1$ and $k T=0.001$ (measured in the units of $D / 50$ ). Differential conductance, $G_{\text {diff }}=\mathrm{d} I / \mathrm{d} V$, is shown in the inset to Fig. 1a for different magnetic configurations (different values of $\theta$ ). Due to the effective exchange field $B_{\text {ex }}$ (proportional to spin polarization of the electrodes [3]), the zero-bias Kondo anomaly is split. The splitting strongly depends on the magnetic configuration and on polarization $p_{\mathrm{L}}=p_{\mathrm{R}}=p$, as displayed in Fig. 1a, where half of the splitting is shown as a function of the angle $\theta$. In the parallel configuration $(\theta=0)$ the splitting considerably increases with the polarization $p$. When $\theta$ increases, the splitting monotonically decreases and vanishes in the antiparallel configuration $(\theta=\pi)$.

The linear conductance $G$ in the Kondo regime (the dot level $E_{0}=-0.35$ ) as a function of $p$ is presented in Fig. $1 \mathrm{~b}$ for three different configurations. As 

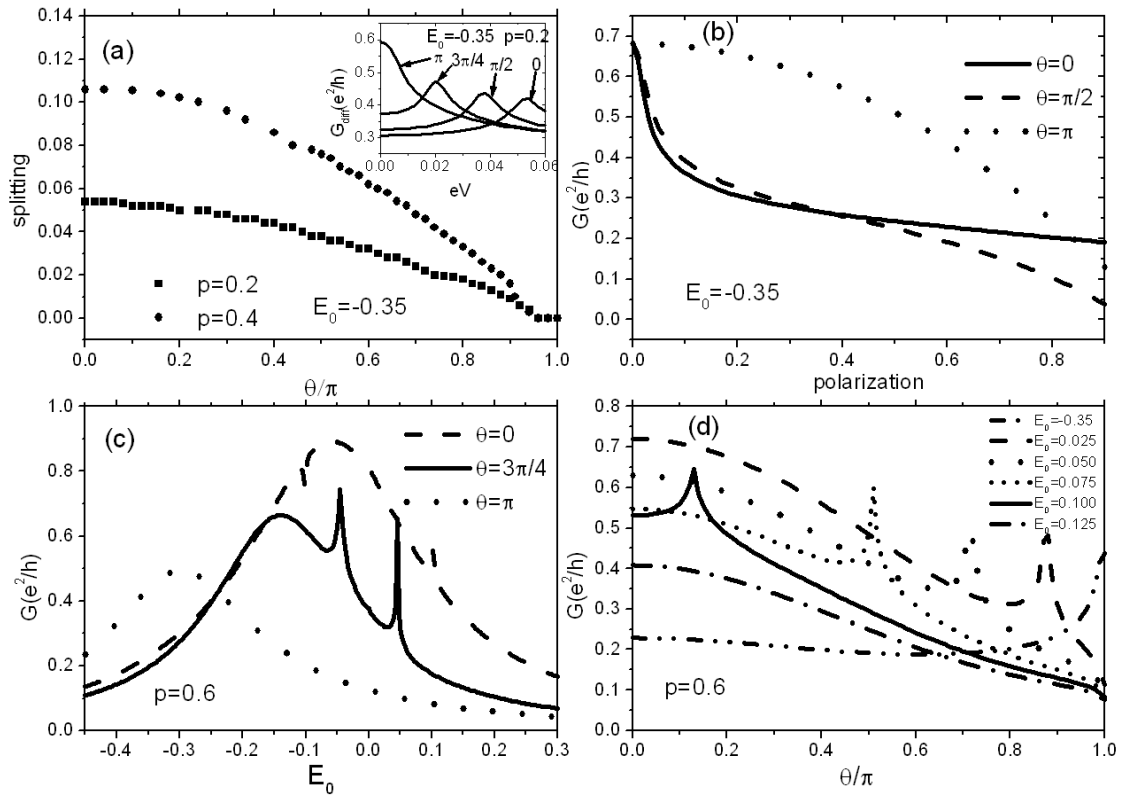

Fig. 1. Half of the Kondo anomaly splitting versus $\theta$ (a) and the linear conductance as a function of polarization (b), energy level $E_{0}(\mathrm{c})$ and the angle $\theta(\mathrm{d})$.

one could expect, the conductance monotonically decreases with increasing polarization, but the character of the changes strongly depends on $\theta$. In the parallel configuration the conductance rapidly decreases in a region of small $p$, while for higher polarization it decreases rather slowly. Such a dependence can be easily understood when the influence of exchange field on the zero-bias Kondo anomaly is taken into account. As $p$ becomes nonzero, the corresponding exchange field leads to a splitting of the zero bias anomaly and consequently to suppression of the Kondo resonance. This leads to a fast drop in conductance shown in Fg. 1b. For higher values of $p$, the changes in conductance are not so pronounced and the linear conductance weakly drops with further increase in $p$.

Quite different behavior takes place for $\theta=\pi$. The changes in conductance are minor in the region of small $p$, but $G$ decreases relatively fast as $p$ tends to 1 . This behavior is a consequence of the absence of exchange field in the antiparallel configuration and therefore absence of the Kondo anomaly splitting. Moreover, the zero-bias conductance becomes suppressed in the antiparallel configuration due to vanishing density of states in the sink (source) electrode for spin-up (spin-down) electrons. When magnetic moments in both electrodes are perpendicular, $\theta=\pi / 2$, changes in the conductance are significant in the limit of small $p$ as well as for $p$ approaching 1.

The linear conductance calculated as a function of the dot level $E_{0}$ reveals a considerable dependence on the magnetic configuration, as shown in Fig. 1c. For 
the assumed polarization, $p=0.6$, the Kondo effect, though considerably reduced, survives in the antiparallel configuration, so the spectrum shows a well-defined maximum in the Kondo regime. When $\theta$ is small, a strong suppression of the conductance is obtained in the Kondo regime due to a large splitting of the anomaly, but the conductance increases and achieves a wide maximum as the dot level approaches the Fermi level in the leads. However, additional features, a dip and a peak or two peaks, appear in the spectrum which result from the presence of $B_{\mathrm{ex}}$. The conductance is enhanced if one of the spin split levels crosses the Fermi energy, whereas it is suppressed for $E_{\mathrm{F}}$ lying in the gap. Such effects lead to a non-monotonic dependence of $G$ versus $\theta$ as depicted in Fig. 1d. When $\left|E_{0}-E_{\mathrm{F}}\right|<$ $\frac{1}{2} \Delta_{0}$, with $\Delta_{0}$ corresponding to the level splitting for $\theta=0$, a pronounced peak can be observed for a certain non-collinear configuration, but the conductance decreases monotonically for $E_{0}-E_{\mathrm{F}}>\frac{1}{2} \Delta_{0}$. A different dependence is obtained in the Kondo regime $\left(E_{0}=-0.35\right)$ where the conductance at first slightly decreases, and then considerably increases reaching a maximum in the antiparallel configuration.

\section{Acknowledgments}

This work was supported by funds of the Polish Ministry of Science and Higher Education as a research project in years 2006-2008 and 2006-2009.

\section{References}

[1] I. Gores, D. Goldhaber-Gordon, S. Heemeyer, M A. Kastner, H. Shtrikman, D. Mahalu, U. Meirav, Phys Rev B 62, 2188 (2000).

[2] J. Martinek, Y. Utsumi, H. Imamura, J. Barnaś, S. Maekawa, J. Konig, G. Schon, Phys. Rev. Lett. 91, 127203 (2003).

[3] R. Świrkowicz, M. Wilczyński, M. Wawrzyniak, J. Barnaś, Phys. Rev. B 73, 193312 (2006).

[4] Y. Meir, N.S. Wingreen, P.A. Lee, Phys. Rev. Lett. 70, 260 (1993).

[5] T.K. Ng, Phys. Rev. Lett. 70, 3635 (1993). 